\title{
Fault Diagnosis on Cabin Electric Power Equipments
}

\author{
Baoji YUAN* \\ College of Navy Submarine \\ Qingdao, Shandong, P.R. China \\ E-mail: 18661780382@163.com \\ +* Corresponding author
}

\author{
Weining LI \\ College of Navy Submarine \\ Qingdao, Shandong, P.R. China \\ E-mail: 1918808552@qq.com
}

\author{
Yanfeng WU \\ College of Navy Submarine \\ Qingdao, Shandong, P.R.China \\ E-mail: 15066792976@163.com
}

\begin{abstract}
Design Integrated construction of fault diagnose system with centralized control in this paper. The diagnose system has been divided into main station and slave station by different functions. We has structured the software frame of diagnose system so as to increase efficient of fault diagnose on voltage changer and breaker based on fault diagnose procedure.
\end{abstract}

Keywords-electric power system; centralized control; fault diagnose; integrated design

\section{INTRODUCTION}

Correlation studies on fault diagnose of electric centralized control[1] are not paid more attention. The cause is that electric centralized control system is considered as a monitoring and executing center, instead of a diagnose and decision-making center[2]. Along with lots of unmanned electric equipments, monitoring and measuring equipments increasing, fault diagnose of electric centralized control system has more prospects for development[3].

\section{INTEGRATED CONSTRUCTION}

The electric centralized control system consists of three integration platforms, concluding electric equipment synthesize information integration platform (electric converting station, power equipment running working condition, monitoring on-line, secondary equipment information, electric network information, etc), network transmission integration platform[4] (unified transmission protocols, avoiding repetition and reconstruction by different subsystems, promoting reliability and transmission ability of transmission platform), application integration platform(constructing monitor workstation, information confluens of several signals including equipment state, video signal, meteorological phenomena, protective action, and oscilloscope data). Based on integrated platforms and database, the functions consisting monitoring safe and prewarning of integrated platform can be realized[5]. Electric centralized control system' structural block diagram is showed in Fig 1.

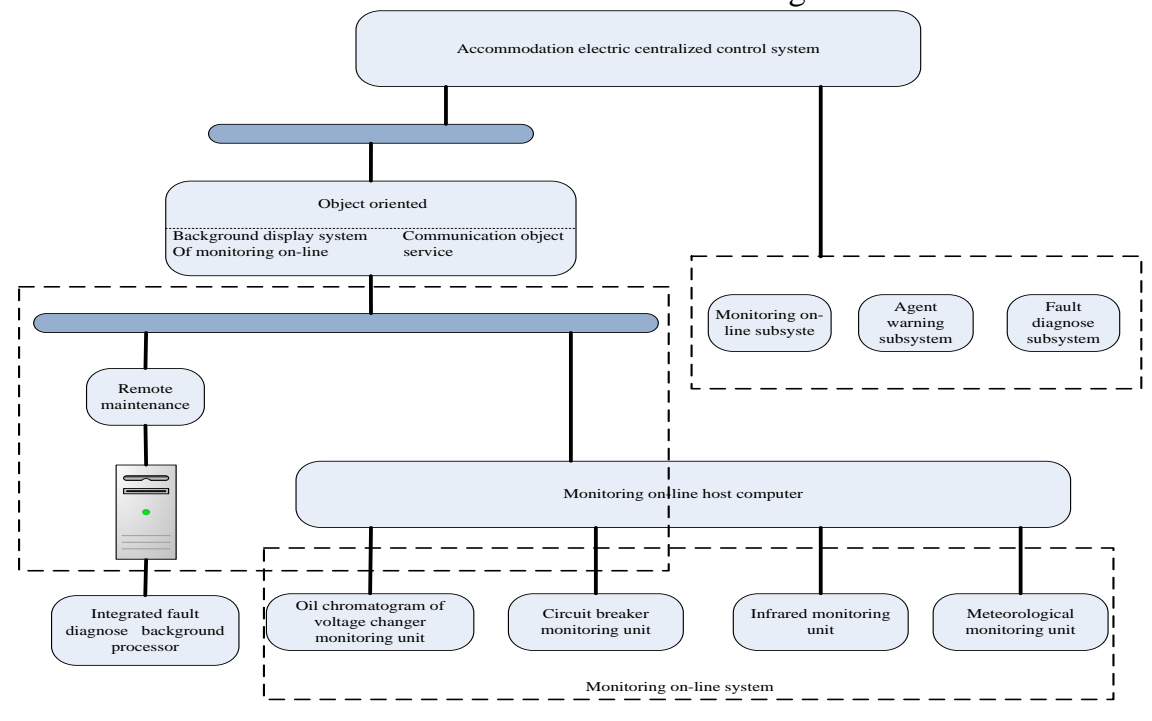

Figure 1. Structural block diagram of accommodation electric centralized control system 


\section{FunCtion MODUlE}

Function modules[6] concludes electric converting station monitoring function, modal manage function, incident and warning function, advanced analysis function, data collection and translation, data handling and storage, system modeling and preventive maintenance.

3.1 Substation function

(1) Data display: Display timely every monitoring unit's working state and every monitoring data's curve.

(2) Data storage: Store up every monitoring unit's data. Store interior data and preliminary information of station.

(3)Remote telecommunication: Data communication with remote manages system at regular time.

3.2 Centralized control item's function

(1) Data collection and handling: Receive and deal with substation's all of data by data communication service integrated platform.

(2) Historical data storage: Record historical data for inspect the system state of warning event.
(3) Monitoring function: Monitor timely all of working data and equipment state of main electric equipments.

(4) Warning function: When the collected electric equipment's voltage or current value exceeds threshold value, it can be self-diagnosed fault signal and handle warning process automatically.

(5) Warning handle function and fault diagnose function: Divide signals from fault substation into different categories and different levels, and put the analysis result return to browser item for display.

(6) Detailed diagnose module: According to warning signals and abnormal signals, define fault cause and give to plan to handle this fault after warning handle module and fault diagnose module giving warning signals and defining the specific fault equipment.

\section{Software Frame of FAult Diagnose}

According to above fault diagnose function's requires, information categories and information features, centralized control fault diagnose software's structural block diagram is showed as follow.

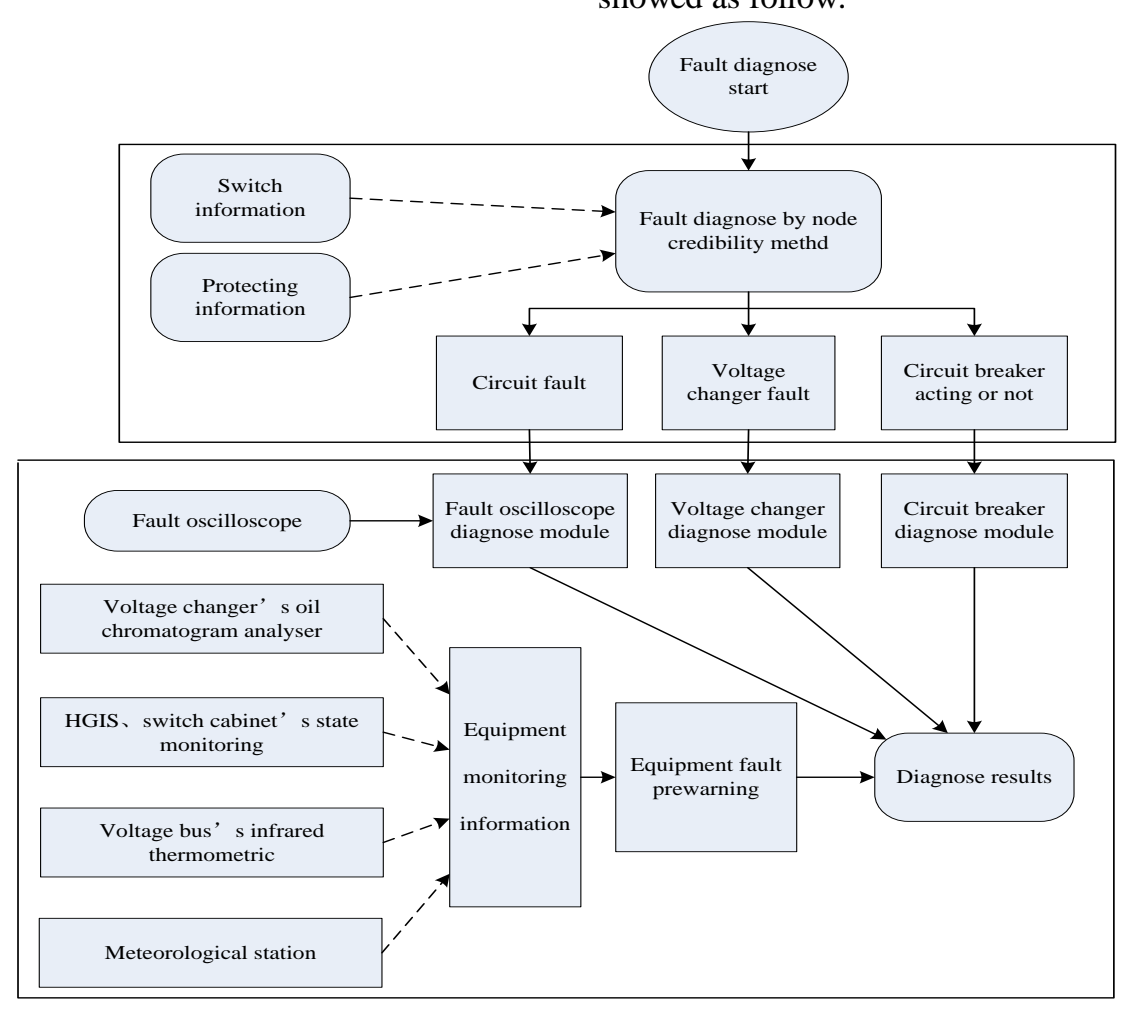

Figure 2. Centralized control fault diagnose software's structural block diagram

Fault diagnose has two steps: Firstly, apply circuit breaker and protective information and rapidly define fault equipment and fault type by node credibility. At the time confirm the trueness of warning signal. Secondly, check up roundly the definitive equipment according to first diagnose result.

\section{A. Integrated Diagnose of Voltage Changer}

We can know working state of voltage changer by analysis of solution-gas[7], and analyze the cause of abnormal phenomenon and fault signal, so as to forecast further information of electric equipment. Further, change term maintenance and repair to fault warning and handling automatically. According to colour spectrum of voltage changer, we can know if voltage changer has fault, fault type, fault severity, and fault variety.

\section{B. Integrated Diagnose of Circuit Breaker}

Based on analyzing elemental state data and collected working parameters of circuit breaker from communication 
integrated platform, we can get characteristic variables. For example, we get circuit switch coils' characteristic information including current's occurrence time, current's extinction time, current's maximum value, maximum value's occurrence time, and coil current's effective value. Combining with expert knowledge base, we can get correct diagnose analysis synthetically by Dempster compound principle.

\section{Abnormal State Monitoring On-line with Wavelet Analysis}

Fourier analysis method only satisfies stationary signal analysis, but non-stationary signals are anywhere in engineering domain. Advanced short-time Fourier conversion is developed for satisfying non-stationary signal analysis, but its window function's size and shape are fixed which do not satisfy different frequency. On practical application, wavelet analysis method can achieve that applies wide window function to satisfy low frequency signal, and applies narrow window function to satisfy high frequency signal with spectral line's high-resolution.

Wavelet analysis method[8] is the way to partially analysis the signal in time scale, and its time window and frequency window can be changed all in time domain. The low frequency part has higher frequency-resolution and lower time-resolution, on the contrary, the high frequency part has lower frequency-resolution and higher time-resolution. So this method extremely satisfies distinguishing the abnormal signal in normal message and brings up the fault component, and it can be addressed as microscope of signal analysis. In accommodation equipments' fault diagnose, wavelet analysis method have favourable effect on electric parameters and state information.

In electric equipment's fault signal analysis, the frequency feature of signal in every time is vital, so advanced method should combine time-domain and frequency-domain to describe the feature of fault signal. Gladly, wavelet analysis method can deal with these problems. It has fixed window area, but the size and shape of time window and frequency window can be changed.

Supposing $\psi(t) \in L^{2}(R), \quad \psi(t) \quad$ is converted to $\psi(w)$ by Fourier transform, $L^{2}(R)$ is square-integrable space. When $\psi(w)$ satisfies the following condition,

$$
C_{\psi}=\int_{R} \frac{\psi(w)}{|w|} d w<\infty
$$

$\psi(t)$ is a elemental wavelet function. Dilating and translating $\psi(w)$, we can get a wavelet array.

Under the continuous condition, wavelet array becomes

$$
\psi_{a, b}(t)=\frac{1}{\sqrt{a}} \psi\left(\frac{t-b}{a}\right), a, b \in R, a \neq 0
$$

Where $a$ is dilating agent, and $b$ is translating agent. The continuous wavelet transform of any function $f(t) \in L^{2}(R)$ is

$$
W_{f}(a, b)=\left\langle f, \psi_{a, b}\right\rangle=a^{-1 / 2} \int_{R} f(t) \psi\left(\frac{t-b}{a}\right) d t
$$

$W_{f}(a, b)$ is described as wavelet transform of $f(t)$.

$a$ and $b$ should be discretized for realizing rapid wavelet transform on computer.

$$
\begin{gathered}
\psi_{j, k}(t)=2^{-j / 2} \psi\left(2^{-j} t-k\right), j, k \in Z \\
\text { V. } \quad \text { CONCLUSIONS }
\end{gathered}
$$

The action of electric integrated control system is import increasingly along with lots of unmanned accommodation electric equipments increasing. This paper has designed accommodation electric centralized control system and constructed centralized control fault diagnose software procedure. In addition, we have discussed every fault diagnose subsystem which are provided with realistic meaning.

\section{REFERENCES}

[1] Ferreira V.H, Zanghi R, Fortes M.Z,et al. A survey on intelligent system application to fault diagnosis in electric power system transmission lines[J]. Electric Power Systems Research,2016,136(7):135-153.

[2] Sun Mingwei, Tong Xiaoyang, Liu Xinyu,et al. A power system fault diagnosis method using temporal Bayesian knowledge bases[J]. Dianwang Jishu/Power System Technology,2014,38(3):715-722.

[3] WANG Lei,CHEN Qing,GAO Hongyu, et al.Model for And Tree in Power System Fault Diagnosis[J]. Automation of Electric Power Systems,2016,40(2):100-106.

[4] Jain M. Babita, Jain Amit, Srinivas M.B,et al. A web based expert system shell for fault diagnosis and control of power system equipment[J]. Proceedings of 2008 International Conference on Condition Monitoring and Diagnosis, CMD 2008, 2007: 1310-1313.

[5] Yang Xiu-Wen, Chen Jie, Zeng Shun-Peng,et al. Application research of continuous wavelet transform in crack fault diagnosis of transmission gear[J]. Proceedings of the International Computer Conference 2006 on Wavelet Active Media Technology and Information Processing, ICWAMTIP 2006,2006: 1161-1166.

[6] Mao Yongfang, Qin Yi, Tang Baoping,et al. Fault diagnosis of bearing based on overcomplete rational wavelet transform[J]. Journal of Vibration, Measurement and Diagnosis,2011,31(5): 626-630.

[7] V.H. Ferreira, R. Zanghi, M.Z. Fortes , et al. A survey on intelligent system application to fault diagnosis in electric power system transmission lines[J]. Electric Power Systems Research,2016,136(7): 135-153.

[8] Li Hui. Impulse response wavelet transform based bearing fault diagnosis[J]. Advanced Research on Industry, Information System and Material Engineering,2011, 204:1018-1021 